\title{
The Perseverative Thinking Questionnaire in Patients with Persecutory Delusions
}

\author{
Emma Černis \\ University of Oxford, $U K$ \\ Graham Dunn \\ Manchester University, $U K$ \\ Helen Startup \\ University of Oxford, and Sussex Partnership, NHS Foundation Trust, Hove, UK \\ David Kingdon and Gail Wingham \\ University of Southampton, UK \\ Nicole Evans and Rachel Lister \\ University of Oxford, UK \\ Katherine Pugh \\ University of Oxford, and Sussex Partnership, NHS Foundation Trust, Hove, UK \\ Jacinta Cordwell and Helen Mander \\ University of Southampton, UK \\ Daniel Freeman \\ University of Oxford, UK
}

Reprint requests to Emma Černis, Oxford Institute of Clinical Psychology Training, The Isis Education Centre, Roosevelt Drive, Warneford Hospital, Warneford Lane, Oxford OX3 7JX, UK. E-mail: emma.cernis@hmc.ox.ac.uk 
Background: Ruminative negative thinking has typically been considered as a factor maintaining common emotional disorders and has recently been shown to maintain persecutory delusions in psychosis. The Perseverative Thinking Questionnaire (PTQ) (Ehring et al., 2011) is a transdiagnostic measure of ruminative negative thinking that shows promise as a "content-free" measure of ruminative negative thinking. Aims: The PTQ has not previously been studied in a psychosis patient group. In this study we report for the first time on the psychometric properties of Ehring et al.'s PTQ in such a group. Method: The PTQ was completed by 142 patients with current persecutory delusions and 273 non-clinical participants. Participants also completed measures of worry and paranoia. A confirmatory factor analysis was performed on the clinical group's PTQ responses to assess the factor structure of the measure. Differences between groups were used to assess criterion reliability. Results: A three lower-order factor structure of the PTQ (core characteristics of ruminative negative thinking, perceived unproductiveness, and capturing mental capacity) was replicated in the clinical sample. Patients with persecutory delusions were shown to experience significantly higher levels of ruminative negative thinking on the PTQ than the general population sample. The PTQ demonstrated high internal reliability. Conclusions: This study did not include test-retest data, and did not compare the PTQ against a measure of depressive rumination but, nevertheless, lends support for the validity of the PTQ as a measure of negative ruminative thinking in patients with psychosis.

Keywords: Rumination, psychosis, schizophrenia, paranoia, Perseverative Thinking Questionnaire, persecutory delusions.

\section{Introduction}

Traditionally, repetitive negative thinking (RNT) has been studied in emotional disorders such as depression (e.g. Spasojević and Alloy, 2001) but in recent years there has been increasing interest in this thinking style influencing psychotic experiences such as paranoia (e.g. Simpson, MacGregor, Cavanagh and Dudley, 2012; Freeman et al., 2015). In this paper we report on psychometric properties of a relatively new measure of RNT. This measure was originally developed in non-clinical (internet and university student) groups and clinical groups comprised primarily of mood disorder and eating disorder patients.

The Perseverative Thinking Questionnaire (PTQ; Ehring et al., 2011) is a self-report measure of negative ruminative thinking (RNT). It is a "content-free" measure, which includes items such as "my thoughts are not much help to me" and "I keep thinking about the same issues all the time". This is of note, since previous measures of rumination, such as the Response Style Questionnaire (RSQ; Nolen-Hoeksema and Morrow, 1991) and the Penn State Worry Questionnaire (PSWQ; Meyer, Miller, Metzger and Borkovec, 1990) use disorderspecific content in their items (in the case of the RSQ and PSWQ, depressive rumination and Generalized Anxiety Disorder content, respectively, is used). The PSWQ is described here as a measure of rumination as well as worry, since worry is a content-specific form of RNT (Topper, Molenaar, Emmelkamp and Ehring, 2014).

Since RNT is a process that has been demonstrated to underlie a number of different disorders, with key characteristics common between disorders, and only the content showing disorder specificity (Ehring and Watkins, 2008), a reliable transdiagnostic measure is a 
valuable tool for research in this area. The PTQ, with its "content-free" items is a promising candidate, but has yet to be validated in a psychosis sample.

Ehring et al. (2011) developed the PTQ using data from non-clinical, mood disorder, and eating disorder groups. During its development, confirmatory factor analyses found a second-order model structure with three lower-order factors. These are described as "Core characteristics" of RNT (e.g. "The same thought keeps going through my mind again and again"); "Perceived unproductiveness" of RNT (e.g. "I think about many problems without solving any of them"), and "Capturing mental capacity" (e.g. "My thoughts prevent me from focusing on other things").

When validating the PTQ, Ehring et al. (2011) called for future research to use different carefully diagnosed groups to validate the PTQ. However, to date, the few studies published that use the PTQ have mainly focused on depression or used non-clinical samples. For example, Raes (2012) demonstrated that the PTQ was a significant predictor of depressive symptoms 3 years later in a sample of 81 university students and Teismann et al. (2014) found that a group therapy aimed at reducing rumination (as measured by the PTQ) improved depressed mood in patients with residual depressive symptoms. Using non-clinical participant groups, Teismann, Het, Grillenberger, Willutzki and Wolf (2013) found that writing about life goals reduced ruminative thinking in a general population sample; and Burg and Michalak (2011) showed that maintaining mindful contact with the breath was negatively correlated with RNT in undergraduate students. Müller, Teismann, Havemann, Michalak and Seehagen (2013) found that antenatal scores on the PTQ assessed in 66 mothers had predictive value for impaired bonding with, and anxiety about caring for, the child. Vossbeck-Elsebusch, Freisfeld and Ehring (2014) found a correlation between new mothers' PTQ scores and their symptoms of post-traumatic stress disorder following childbirth.

Whilst Martinelli, Cavanagh and Dudley (2013) have used the PTQ in a non-clinical sample to investigate the role of rumination in the maintenance of non-clinical paranoia, to our knowledge there has been no previous work using the PTQ in a clinical population with psychosis diagnoses. However, patients with current persecutory delusions have already been described as demonstrating clinical levels of rumination (i.e. comparative to scores of Generalised Anxiety Disorder patients) using the ruminative response scale of the Response Styles Questionnaire (Hepworth, Startup and Freeman, 2011) and worry using the PSWQ (e.g. Freeman, Pugh, Vorontsova, Antley and Slater, 2010). This is in keeping with Freeman, Garety, Kuipers, Fowler and Bebbington's (2002) cognitive model of persecutory delusions, which outlines worry as a key mechanism in the development and maintenance of the delusion. This model has been supported in many studies; most notably, Freeman et al. (2015) demonstrated in a randomized controlled trial with 150 patients with persistent persecutory delusions that a brief CBT intervention targeting worry (a form of rumination) led to significant reductions in paranoia. A sophisticated mediation analysis showed that two-thirds of the changes in the persecutory delusions were accounted for by changes in worry. Significant changes in RNT as assessed by the PTQ were also found in the trial. This trial provides the strongest demonstration yet of a causal role of worry and paranoia. Using a non-clinical sample, Simpson et al. (2012) found associations between trait paranoia and rumination in a non-clinical sample. It would therefore be beneficial to validate the structure of the PTQ in this patient group in order to assess whether it may be a reliable and valid measure of RNT for this population. 
The current study therefore seeks to validate the PTQ by assessing its factor structure in this clinical population and by using the measure to establish criterion validity in comparison of a population of patients with current persecutory delusions against a sample from the general population. It was also planned to test the PTQ's internal reliability, as well as its convergent validity, with an existing rumination measure frequently used with this population: the PSWQ.

Since the PTQ is intended to be a measure of a transdiagnostic process, we hypothesize that its factor structure will be replicated in patients with persecutory delusions, as RNT in the form of worry is implicated in the maintenance of persecutory delusions. It is also predicted that a valid measure of RNT would show significant differences between the levels of perseverative thinking in this group in comparison to a general population (non-clinical) group (i.e. will show criterion validity). We predicted demonstration of convergent validity with the PSWQ. Further, since worry is a form of RNT that has been implicated in the development and maintenance of paranoia (Freeman et al., 2002) and demonstrated to correlate with paranoia using the PSWQ (e.g. Freeman et al., 2008), we also predicted that PTQ scores will correlate with paranoia scores.

\section{Method}

\section{Participants}

One hundred and forty-two patients with persecutory delusions completed the PTQ during the baseline assessment, prior to randomization, of a clinical trial (ISRCTN23197625) (Freeman et al 2012; Freeman et al., 2015). The participants were recruited from two mental health NHS Trusts: Oxford Health NHS Foundation Trust and Southern Health NHS Foundation Trust.

The inclusion criteria were: a current persecutory delusion as defined by Freeman and Garety (2000); scoring at least 3 on the conviction scale of the PSYRATS delusions scale (i.e. at least 50\% conviction in the delusion) (Haddock, McCarron, Tarrier and Faragher, 1999); that the delusion had persisted for at least 3 months; a clinical diagnosis of schizophrenia, schizoaffective disorder or delusional disorder; a clinically significant level of worry, as indicated by scores above 44 on the Penn State Worry Questionnaire (see Startup and Erickson, 2006); aged between 18 and 65; and no changes in medication in the past month. Criteria for exclusion were: a primary diagnosis of alcohol or substance dependency or personality disorder; organic syndrome or learning disability; a command of spoken English inadequate for engaging in therapy; and currently having individual CBT.

The non-clinical group was made up of 273 volunteers from the general population who filled in the measures via online survey software (Bristol Online Survey) during screening for suitability for other studies run by the Oxford Cognitive Approaches to Psychosis research group (Freeman et al., 2014).

\section{Measures}

Perseverative Thinking Questionnaire (PTQ; Ehring et al., 2011). The PTQ is a 15-item measure of repetitive negative thinking. Each item is rated on a 5-point scale, from $0=$ "Never" to $4=$ "almost always" and asks participants to indicate how they typically think about negative experiences or problems. Scores can range from 0 to 60, and higher scores indicate higher levels of repetitive thought. 
Penn State Worry Questionnaire (PSWQ; Meyer et al., 1990). The PSWQ is the most established measure of trait worry style ("I have been a worrier all my life") and has been used in non-clinical and clinical populations (see review by Startup and Erickson, 2006). Each of the 16 items is rated on a 5-point scale. Higher scores indicate a greater tendency worry.

Green et al. Paranoid Thoughts Scale (GPTS; Green et al., 2008). The GPTS is a 32item measure of paranoid thinking. Part A assesses ideas of reference (e.g. "It was hard to stop thinking about people talking about me behind my back") and Part B assesses ideas of persecution (e.g. "I was convinced there was a conspiracy against me"). Each item is rated on a 5-point scale. Higher scores indicate greater levels of paranoid thinking. The scale was completed for the period of the previous fortnight. The non-clinical group completed Part B only.

\section{Analysis}

The confirmatory factor analysis (CFA) was carried out using Mplus Version 7 (Muthén and Muthén, 1998-2012). All other analyses were carried out using SPSS Version 20 (IBM, 2011) and all hypothesis testing was two-tailed.

First, the internal reliability of the PTQ was assessed by calculating Cronbach's alphas for each participant group. Next, a CFA for the patient group was performed to test for the proposed second order model of the PTQ: with one higher-order and three lower-order factors. This was assessed using chi-square $\left(\chi^{2}\right)$ and Comparative Fit Index (CFI) statistics. With this approach, a good fit is indicated by non-significant chi-square statistics and CFI greater than 0.95. Then, PTQ scores for both participant groups were compared using $t$-tests to establish criterion validity.

Finally, convergent validity of the PTQ was assessed by correlating PTQ total scores for each participant group with their PSWQ total scores and with GPTS Part B scores.

\section{Results}

\section{Demographics of the sample}

Basic demographic and clinical data for both the clinical and non-clinical participant groups are displayed in Table 1. Analysis with a $t$-test indicated no significant difference in age, and a chi-square test indicated no significant difference in ethnicity between the two groups, but there were proportionally more males in the clinical than the non-clinical group $\left(\chi^{2}=20.11\right.$, $p<.001)$.

\section{Internal reliability of scale}

Cronbach's alphas were calculated for each group to determine the internal reliability of the PTQ measure. Internal reliability was found to be high (Clinical: Cronbach's alpha $=0.923$; Non-clinical: Cronbach's alpha $=0.968$ ). 
Table 1. Demographics of the two participant groups, including clinical details for the clinical group. Analysis indicates no significant differences between groups in age or ethnicity, but the clinical group was found to include proportionally more male participants than the non-clinical group.

\begin{tabular}{|c|c|c|}
\hline & Clinical & Non-clinical \\
\hline$\overline{\text { Age (years) }}$ & $41.5(11.4)$ & $40.1(15.4)$ \\
\hline \multicolumn{3}{|l|}{ Mean $(S D)$} \\
\hline \multirow{4}{*}{$\begin{array}{l}\text { Gender: } \\
\text { Male: Female }\end{array}$} & $82: 60$ & $95: 178$ \\
\hline & & \\
\hline & 129 White & 250 White \\
\hline & 1 Black & 3 Black \\
\hline \multirow[t]{3}{*}{ Ethnicity } & 3 Indian & 3 Indian \\
\hline & 2 Chinese & 4 Chinese \\
\hline & 7 Other & 12 Other \\
\hline \multirow[t]{6}{*}{ Current employment status } & 13 Full-time employed & 110 Full-time employed \\
\hline & 14 Part-time employed & 38 Part-time employed \\
\hline & 103 Unemployed & 28 Unemployed \\
\hline & 7 Retired & 32 Retired \\
\hline & 2 Student & 41 Student \\
\hline & 3 Self-employed & 24 Self-employed \\
\hline \multirow[t]{4}{*}{ Diagnosis } & 104 Schizophrenia & - NA - \\
\hline & 11 Schizoaffective Disorder & \\
\hline & 9 Delusional Disorder & \\
\hline & 18 Psychosis NOS & \\
\hline $\begin{array}{l}\text { Antipsychotic Medication Dose } \\
\text { (Equivalent to Cholorpromazine, mg) } \\
\text { Mean }(S D)\end{array}$ & $495.0(412.3)$ & - NA - \\
\hline
\end{tabular}

Factor structure of the $P T Q$

The data from the 142 participants in the clinical group were analysed using a confirmatory factor analysis. Traditional CFA indicated a good fit of the data to Ehring et al.'s (2011) factor structure of one higher-order and three lower-order factors $\left(\chi^{2}=332.40, d f=87, \mathrm{CFI}=\right.$ 0.96). Note that this CFI is comparable to that of Ehring et al.'s clinical sample, study 1 $(\mathrm{CFI}=0.95)$.

\section{Comparison of patients with the non-clinical controls}

The total PTQ score and each of the three lower-order subscale scores were found to be significantly higher in the clinical than the non-clinical group. The results for these $t$-tests are shown in Table 2. For all comparison, Levene's tests of equal variance indicated that the standard deviations of the two groups could not be assumed to be equal, and therefore $t$-tests based on a Satterthwaite approximation were used.

The PTQ total scores for both groups are consistent with previous research: Martinelli et al. (2013) report a mean total of $28.91(S D=11.77)$ on the PTQ in their student sample, whilst Raes' (2012) and Burg and Michalak's (2011) student groups scored a mean of 28.85 $(S D=8.31)$ and $26.78(S D=9.57)$ respectively. The clinical group here score similarly to 
Table 2. Showing the statistics for the $t$-tests carried out to test for statistically significant differences between the two groups' scores on the PTQ (total and subscales)

\begin{tabular}{lllllll}
\hline & $\begin{array}{l}\text { Clinical }(n= \\
\text { 142) }\end{array}$ & $\begin{array}{l}\text { Non-clinical } \\
(n=273)\end{array}$ & & & \\
Variable & Mean $(S D)$ & Mean $(S D)$ & \multicolumn{1}{l}{$t$} & $d f$ & $\boldsymbol{p}$ \\
\hline PTQ total score & $44.6(9.7)$ & $26.1(13.6)$ & -16.0 & 373.7 & $<.001$ \\
Core characteristics subscale & $27.8(5.9)$ & $17.3(8.5)$ & -14.7 & 377.8 & $<.001$ \\
Perceived unproductiveness subscale & $8.4(2.5)$ & $4.8(3.0)$ & -12.8 & 331.0 & $<.001$ \\
Mental capacity subscale & $8.4(2.4)$ & $4.0(3.0)$ & -16.2 & 338.5 & $<.001$ \\
\hline
\end{tabular}

Teismann et al.'s (2014) two depression groups (baseline results for active group: mean = 41.32, $S D=8.80$; baseline for control group: mean $=43.11, S D=7.66$ ).

\section{Convergent validity}

The convergent validity between the PTQ and PSWQ was found to be high for each participant group (Non clinical: $r=0.79, p<.001$; Clinical: $r=0.50, p<.001$ ).

Convergent validity with the GPTS Part B was also significant (Nonclinical: $r=0.457$, $p<.001$; Clinical: $r=0.299, p<.001)$.

\section{Discussion}

The current study sought to validate Ehring et al.'s Perseverative Thinking Questionnaire (PTQ) in a different patient group: people with persecutory delusions. Validating the PTQ in this group would support Ehring et al.'s view that the PTQ is likely to be a transdiagnostic, "content-free" measure of repetitive negative thinking (RNT), which is proposed to maintain a range of psychological disorders. The one higher-order, three lower-order factor structure of the PTQ has been confirmed here, with values comparable to those in Ehring et al.'s (2011) validation of the measure.

Analysis has also shown the ability of the PTQ to distinguish between a clinical and nonclinical sample, demonstrating its criterion validity. The clinical group scored significantly higher than the non-clinical participants on every subscale of the measure.

The results of the current study lend support to the theoretical conceptualization of the PTQ as a transdiagnostic measure of RNT. By replicating the factor structure with a novel clinical group, the assertion that the PTQ measures a transdiagnostic process is supported; whilst finding significant differences in PTQ scores between groups of participants with and without a clinical symptom theorised to be maintained by worry (a ruminative process) establishes the validity of the PTQ. The convergent validity of the PTQ with the PSWQ is also positive, as it suggests that the PTQ is measuring sequelae also assessed by the PSWQ, thus supporting its validity as a measure of RNT.

A further strength of the PTQ is its relative ease of use for patients, particularly in comparison to the PSWQ, which includes a number of fairly complex-worded reverse items. The PTQ uses items that are short and easily understood, with no reversed items. This is a 
benefit when working with a psychosis population as cognitive difficulties are common in this group and therefore more accessible questionnaires are preferable.

\section{Limitations}

There are limitations to the current study that should be addressed in further evaluation of the PTQ in a psychosis population. First, we have not completed a test-retest reliability analysis. This would be a valuable addition to future research in this area.

A further weakness of the study is that the PTQ was only compared against the PSWQ and not a standard depressive rumination measure, which would have offered a more complete understanding of the value of the PTQ in comparison to existing measures of RNT.

It could also be argued that the clinical sample used in this study is not representative, since the clinical trial they were being assessed for has an entry criterion of a high PSWQ score. This therefore selects the highest worriers in the population and it would naturally follow that these participants would show high scores on the PTQ, since worry and RNT are related concepts. However, in the recruitment of 150 participants with a persecutory delusion for the clinical trial, only 8 people were excluded on the basis of scoring below cut-off on the PSWQ. Hence it could be said that high worry is a typical feature in the overwhelming majority of people with current persecutory delusions, and therefore that this high-worry sample is representative. Nevertheless, future research may wish to consider using a comparison group of patients without persecutory delusions. Overall, this study supports the use of the PTQ in psychosis populations.

\section{Acknowledgements}

This study was carried out within a project (09/160/06) awarded by the Efficacy and Mechanism Evaluation (EME) Programme, and is funded by the UK Medical Research Council (MRC) and managed by the UK NHS National Institute for Health Research (NIHR) on behalf of the MRC-NIHR partnership. DF is supported by a UK Medical Research Council (MRC) Senior Clinical Fellowship (G0902308). Methodological research by GD is supported by the MRC (MR/K006185/1).

Ethical standards: The authors assert that all procedures contributing to this work comply with the ethical standards of the relevant national and institutional committees on human experimentation and with the Helsinki Declaration of 1975, as revised in 2008.

Conflict of interest: The authors have no conflicts of interest with respect to this publication.

\section{References}

Burg, J.M. and Michalak, J. (2011). The healthy quality of mindful breathing: associations with rumination and depression. Cognitive Therapy and Research, 35, 179-185.

Ehring, T. and Watkins, E.R. (2008). Repetitive negative thinking as a transdiagnostic process. International Journal of Cognitive Psychotherapy, 1, 192-205. 
Ehring, T., Zetsche, U., Weidacker, K., Wahl, K., Schönfeld, S. and Ehlers, A. (2011). The Perseverative Thinking Questionnaire (PTQ): validation of a content-independent measure of repetitive negative thinking. Journal of Behavioral Therapy and Experimental Psychiatry, 42, 225232.

Freeman, D., Dunn, G., Murray, R.M., Evans, N., Lister, R., Antley, A., et al. (2014). How cannabis causes paranoia: using the intravenous administration of $\Delta^{9}$-tetrahydrocannabinol (THC) to identify key cognitive mechanisms leading to paranoia. Schizophrenia Bulletin. Published online before print 15 July 2014, doi: 10.1093/schbul/sbu098

Freeman, D., Dunn, G., Startup, H. and Kingdon, D. (2012). The effects of reducing worry in patients with persecutory delusions: study protocol for a randomized controlled trial. Trials, 13, 223.

Freeman, D., Dunn, G., Startup, H., Pugh, K., Cordwell, J., Mander, H., et al. (2015) Effect of cognitive behaviour therapy for worry on persecutory delusions in patients with psychosis (WIT): a parallel, single-blind, randomised controlled trial with a mediation analysis. The Lancet Psychiatry, 2, 305-313.

Freeman, D. and Garety, P.A. (2000). Comments on the content of persecutory delusions: does the definition need clarification? British Journal of Clinical Psychology, 39, 407-414.

Freeman, D., Garety, P.A., Kuipers, E., Fowler, D. and Bebbington, P.E. (2002). A cognitive model of persecutory delusions. British Journal of Clinical Psychology, 41, 331-347.

Freeman, D., Pugh, K., Antley, A., Slater, M., Bebbington, P., Gittins, M., et al. (2008). Virtual reality study of paranoid thinking in the general population. British Journal of Psychiatry, 192, 258263.

Freeman, D., Pugh, K., Vorontsova, N., Antley, A. and Slater, M. (2010). Testing the continuum of delusional beliefs: an experimental study using virtual reality. Journal of Abnormal Psychology, 119, 83-92.

Green, C., Freeman, D., Kuipers, E., Bebbington, P., Fowler, D., Dunn, G., et al. (2008). Measuring ideas of persecution and reference: the Green et al. Paranoid Thought Scales (G-PTS). Psychological Medicine, 38, 101-111.

Haddock, G., McCarron, J., Tarrier, N. and Faragher, F. B. (1999). Scales to measure dimensions of hallucinations and delusions: the psychotic symptom rating scales (PSYRATS). Psychological Medicine, 29, 879-889.

Hepworth, C., Startup, H. and Freeman, D. (2011). Developing treatments of persistent persecutory delusions: the impact of an emotional processing and metacognitive awareness intervention. The Journal of Nervous and Mental Disease, 199, 653-658.

IBM (2011). SPSS Statistics Version 20. Release 20.0.0. IBM Corporation.

Martinelli, C., Cavanagh, K. and Dudley, R.E.J. (2013). The impact of rumination on state paranoid ideation in a nonclinical sample. Behavior Therapy, 44, 385-394.

Meyer, T.J., Miller, M.L., Metzger, R.L. and Borkovec, T.D. (1990). Development and validation of the Penn State Worry Questionnaire. Behaviour Research and Therapy, 28, 487-495.

Müller, D., Teismann, T., Havemann, B., Michalak, J. and Seehagen, S. (2013). Ruminative thinking as a predictor of perceived postpartum mother-infant bonding. Cognitive Therapy and Research, 37, 89-96.

Muthén, L.K. and Muthén, B.O. (1998-2012). MPlus User's Guide (7th edn.) Los Angeles, CA: Muthén and Muthén.

Nolen-Hoeksema, S. and Morrow, J. (1991). A prospective study of depression and posttraumatic stress symptoms after a natural disaster: the 1989 Loma Prieta earthquake. Journal of Personality and Social Psychology, 61, 115-121.

Raes, F. (2012). Repetitive negative thinking predicts depressed mood at 3-year follow-up in students. Journal of Psychopathology and Behavioral Assessment, 34, 497-501.

Simpson, J., MacGregor, B., Cavanagh, K. and Dudley, R.E.J. (2012). Safety behaviours, rumination and trait paranoia in a non-clinical sample. Journal of Experimental Psychopathology, 3, 612-623. 
Spasojević, J. and Alloy, L.B. (2001). Rumination as a common mechanism relating depressive risk factors to depression. Emotion, 1, 25-37.

Startup, H.M. and Erickson, T.M. (2006). The Penn State Worry Questionnaire (PSWQ). In G.C.L. Davey and A. Wells (Eds), Worry and its Psychological Disorders (pp 101-120). Chichester: Wiley.

Teismann, T., Het, S., Grillenberger, M., Willutzki, U. and Wolf, O.T. (2013). Writing about life goals: effects on rumination, mood and the cortisol awakening response. Journal of Health Psychology. Published online before print July 1 2013, doi:10.1177/1359105313490774

Teismann, T., von Brachel, R., Hanning, S., Grillenberger, M., Hebermehl, L., Hornstein, I., et al. (2014). A randomized controlled trial on the effectiveness of a rumination-focused group treatment for residual depression. Psychotherapy Research, 24, 80-90.

Topper, M., Molenaar, D., Emmelkamp, P. and Ehring, T. (2014). Are rumination and worry two sides of the same coin? A structural equation modelling approach. Journal of Experimental Psychopathology, 5, 363-381.

Vossbeck-Elsebusch, A.N., Freisfeld, C. and Ehring, T. (2014). Predictors of posttraumatic stress symptoms following childbirth. BMC Psychiatry, 14, 200. 